\title{
Sailing through the big crunch-big bang transition
}

\author{
Itzhak Bars, ${ }^{1}$ Paul Steinhardt, ${ }^{2}$ and Neil Turok ${ }^{3}$ \\ ${ }^{1}$ Department of Physics and Astronomy, University of Southern California, Los Angeles, CA, 90089-0484, USA, \\ ${ }^{2}$ Physics Department, Princeton University, Princeton NJ08544, USA, \\ ${ }^{3}$ Perimeter Institute for Theoretical Physics, Waterloo, ON N2L 2Y5, Canada.
}

(Dated: November 20, 2013)

\begin{abstract}
In a recent series of papers, we have shown that theories with scalar fields coupled to gravity (e.g., the standard model) can be lifted to a Weyl-invariant equivalent theory in which it is possible to unambiguously trace the classical cosmological evolution through the transition from big crunch to big bang. The key was identifying a sufficient number of finite, Weyl-invariant conserved quantities to uniquely match the fundamental cosmological degrees of freedom across the transition. In so doing we had to account for the well-known fact that many Weyl-invariant quantities diverge at the crunch and bang. Recently, some authors rediscovered a few of these divergences and concluded based on their existence alone that the theories cannot be geodesically complete. In this note, we show that this conclusion is invalid. Using conserved quantities we explicitly construct the complete set of geodesics and show that they pass continuously through the big crunch-big bang transition.
\end{abstract}

In the standard big bang inflationary mode ${ }^{1}$, the cosmic singularity problem is left unresolved and the cosmology is geodesically incomplete. Consequently, the origin of space and time and the peculiar, exponentially fine-tuned initial conditions required to begin inflation 213 are not explained. In a recent series of papers 4 , we have shown how to construct the complete set of homogeneous classical cosmological solutions of the standard model coupled to gravity, in which the cosmic singularity is replaced by a bounce: the smooth transition from contraction and big crunch to big bang and expansion. These are generic geodesically complete solutions that can, for example, naturally incorporate the cyclic theory of the universe $\mathrm{e}^{12 / 13}$ in which it is proposed that large-scale smoothness, flatness and nearly scale-invariant perturbations are generated during the periods of slow contraction preceding each big bang.

The key to our construction of classical geodesically complete solutions was to "lift" the action (e.g., the standard model coupled to Einstein gravity) to a Weylinvariant equivalent theory. We then identified a number of Weyl-invariant finite quantities ${ }^{7}$ that are conserved near cosmological singularities for symmetry reasons. Our proposal was to match these quantities across the singularities which separate the patches of spacetime describing the big crunch-big bang transition. We showed there were sufficiently many such conserved quantities to ensure a unique match for all cosmological fields. In so doing, we necessarily had to pay attention to the well-known fact $\frac{14}{17}$ that many Weyl-invariant quantities diverge at the bounce, such as the Weyl curvature, $C_{\nu \lambda \delta}^{\mu}$. Recently, Carrasco, Chemisanny and Kallosh 18 and Kallosh and Linde ${ }^{19}$ rediscovered some of these divergences and, without paying attention to our discussion of conserved Weyl-invariant finite quantities, claimed the divergences necessarily spoil the geodesic completeness of our proposed big crunch-big bang transition. In this note, we demonstrate that this naive claim is incorrect.

To be sure, what is presented here is a straightforward elaboration of what was already proven in our ear- lier papers 4 -11. Once we identified a sufficient number of conserved finite Weyl- invariant quantities to determine a unique continuation of all the fundamental cosmological fields (e.g., scalar fields and metric) across all patches of field space, it should be obvious that the spacetime is geodesically complete because the geodesics of particles in the theory are all expressed in terms of these cosmological fields, as detailed below. It remains true that there exist infinitely many Weyl-invariant quantities that diverge at the crunch or bang, but these are irrelevant to the geodesic completeness. In fact, even for these quantities, the field continuation proposed in our papers uniquely determines their evolution before and after they go singular.

To illustrate the point, we focus on the vicinity of the big crunch or big bang where it suffices to consider a simplified standard model with a Higgs-like scalar field conformally coupled to gravity plus radiation. Following the procedure in 411], the "lifted" action of the standard model is achieved by adding an extra scalar field $\phi$ and imposing Weyl symmetry so the number of gauge invariant physical degrees of freedom remain the same. What is achieved in this way is the inclusion of all patches of the fields that leads to a geodesically complete cosmology as explained in more detail in 1011. The leading contributions near the big crunch or the big bang $\operatorname{are}^{7}$ :

$$
\int d^{4} x \sqrt{-g}\left[\frac{1}{2}\left((\partial \phi)^{2}-(\partial h)^{2}\right)+\frac{1}{12}\left(\phi^{2}-h^{2}\right) R\right] .
$$

where $h^{2} \equiv H^{\dagger} H$ for Higgs doublet $H$. The complete description also includes a term describing the radiation (see below). All other contributions to the action, including matter fields, as well as density perturbations become negligible in this limit and the cosmic evolution becomes smoothly ultralocal, meaning that spatial gradients become dynamically negligible ${ }^{20}$. The fact that the spacetime may be treated as spatially homogeneous near the singularity also allows us to find all of its geodesics.

The action is invariant under the local gauge transformations $g_{\mu \nu} \rightarrow \Omega^{-2}\left(x^{\mu}\right) g_{\mu \nu}, \phi \rightarrow \Omega\left(x^{\mu}\right) \phi$ and $h \rightarrow$ 
$\Omega\left(x^{\mu}\right) h$. Although the lift introduces a second scalar field $\phi$ with wrong-sign kinetic energy, it is obviously a gauge-artifact since fixing a Weyl gauge in which $\phi_{E} \equiv$ $(\sqrt{6} / \kappa) \cosh \kappa \sigma / \sqrt{6}$ and $h_{E} \equiv(\sqrt{6} / \kappa) \sinh \kappa \sigma / \sqrt{6}$, where ( $\kappa^{2} \equiv 8 \pi G$, with $G$ Newton's constant) converts the action to Einstein gravity plus canonical scalar field $\sigma$ with no ghost degrees of freedom in the incoming and outgoing cosmological states. Using the Bianchi I, VIII or IX metrics including anisotropy, the line element near the big crunch and bang is: ${ }^{7}$

$$
\begin{aligned}
d s^{2}= & a^{2}(\tau)\left[-d \tau^{2}+e^{-\sqrt{8 / 3} \kappa \alpha_{1}} d \sigma_{3}^{2}+\right. \\
& \left.e^{\sqrt{2 / 3} \kappa \alpha_{1}}\left(e^{\sqrt{2} \kappa \alpha_{2}} d \sigma_{1}^{2}+e^{-\sqrt{2} \kappa \alpha_{2}} d \sigma_{2}^{2}\right)\right]
\end{aligned}
$$

where $\tau$ is the conformal time and $\alpha_{1,2}(\tau)$ parameterize the anisotropy. The $d \sigma_{1,2,3}$ generically include information about the spatial curvature; however, since the spatial curvature is negligible near a big crunch or big bang, $d \sigma_{1,2,3}$ reduce locally to $d x_{1,2,3}$, respectively, resulting in the Kasner-type metric.

Another useful gauge choice, dubbed $\gamma$-gauge fixes $g_{\gamma}=-1$ or equivalently the scale factor $a_{\gamma}=1$, in which case the action specialized to homogeneous fields is:

$$
\int d \tau\left(\frac{1}{2 e}\left[-\dot{\phi}_{\gamma}^{2}+\dot{h}_{\gamma}^{2}+\frac{\kappa^{2}}{6}\left(\phi_{\gamma}^{2}-h_{\gamma}^{2}\right)\left(\dot{\alpha}_{1}^{2}+\dot{\alpha}_{2}^{2}\right)\right]-e \rho_{r}\right)
$$

where $e(\tau)$ is the lapse function and the radiation density is $\rho_{r} / a^{4}(\tau)$ where $\rho_{r}$ is constant.

In this gauge, it is straightforward to find the complete set of solutions that continuously track the evolution of $\phi_{\gamma}, h_{\gamma}$ and $\alpha_{1,2}$ through a big crunch, a brief interlude of antigravity, and then a big bang. Expressing the solution in terms of Einstein gauge fields (indicated by the subscript $E$ ) we obtain ${ }^{7}$ :

$$
\begin{aligned}
a_{E}^{2}(\tau)= & 2|\tau|\left|p+\rho_{r} \tau\right| \\
\alpha_{1,2}(\tau)= & \frac{p_{1,2}}{2 p} \ln \left|\frac{\tau}{T_{1,2}\left(p+\rho_{r} \tau\right)}\right| \\
a_{E}^{2} h_{E}^{2}= & \frac{1}{2}|\tau|\left|p+\rho_{r} \tau\right| \times \\
& \left(\left|T_{3}\left(\rho_{r}+\frac{p}{\tau}\right)\right|^{p_{3} / 2 p}-\left|T_{3}\left(\rho_{r}+\frac{p}{\tau}\right)\right|^{-p_{3} / 2 p}\right)^{2}
\end{aligned}
$$

where $\tau$ is conformal time in units where $\kappa=\sqrt{6}$ and $T_{1,2,3}$ are integration constants. Note that the solution for $a^{2} h^{2}=a_{E}^{2} h_{E}^{2}$ given above is Weyl invariant. The crunch occurs at $\tau_{c}=-p / \rho_{r}$ and the bang at $\tau=0$ with the period of antigravity in between. The constants $p_{1,2,3}$ are the finite conserved values of the canonical momenta of the fields at the crunch or bang: $\pi_{3}=a_{E}^{2} \dot{h}_{E} \rightarrow p_{3}$, $\pi_{1,2}=a_{E}^{2} \dot{\alpha}_{1,2} \rightarrow p_{1,2}$, and $p \equiv \sqrt{p_{1}^{2}+p_{2}^{2}+p_{3}^{2}}$. A $\left|p_{3}\right|$ which is larger than $\sqrt{15}\left(p_{1}^{2}+p_{2}^{2}\right)^{1 / 2}$ insures the avoidance of the mixmaster behavior 2122 even when spatial curvature is present ${ }^{6}$. In Ref. [7, we describe a total of 15 conserved Noether charges that are finite at the crunch or bang and whose conservation across the singularities uniquely determines the solutions given above.

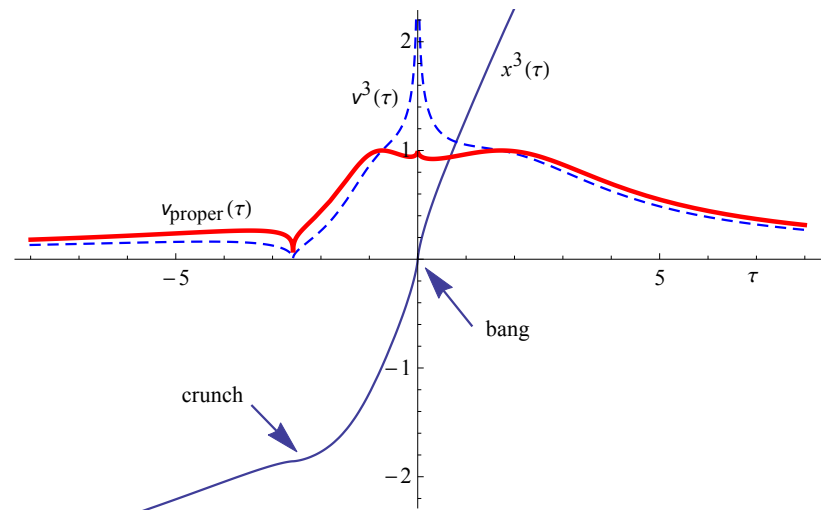

Figure 1. A typical massive particle geodesic, showing the continuous passage through the big crunch and big bang singularities. In this example, the coordinate velocity $v^{3}=\dot{x}^{3}$ goes to zero at the crunch and infinity at the bang. The proper speed of the particle with respect to particles comoving with the $x^{i}$ coordinates, $v_{\text {proper }}=\sqrt{g_{3 i j} \dot{x}^{i} \dot{x}^{j}} / \sqrt{g_{3 i j} \dot{x}^{i} \dot{x}^{j}+m^{2} a^{2}}$, never exceeds unity and touches zero at the crunch and unity at the bang. Likewise, the coordinate $x^{3}$ is finite and continuous throughout. The numerical values of the parameters used to generate this plot are $p_{1}=-1 / 4$, $p_{2}=0, p_{3}=1, T_{1}=1, T_{2}=1, T_{3}=1, k_{1}=0, k_{2}=0, k_{3}=1$, $g_{p}=1, \rho_{r}=0.4$.

We now consider the geodesics of massive particles in the standard model in this geometry. The Weyl-invariant action for a particle moving in a gravitational background can be expressed as:

$$
\mathcal{S}_{\text {particle }}=-\int d \lambda m(x) \sqrt{-\dot{x}^{\mu} \dot{x}^{\nu} g_{\mu \nu}(x)},
$$

where $x^{\mu}(\lambda)$ is a function of the affine parameter $\lambda$. Note that $m(x)$ is generally $x$-dependent in theories like the standard model when the Higgs field contributes to the mass of particles: $m(x)=g_{p} h(x)$, where $g_{p}$ is a dimensionless coupling of the particle field to the Higgs field. From this action, an explicit expression for all geodesics in an anisotropic Kasner universe can be derived, exploiting the spatial homogeneity of the metric $\frac{[5] 10}{}$ :

$$
x^{i}(\tau)=q^{i}+\int^{\tau} d \tau^{\prime} \frac{g_{3}^{i j}\left(\tau^{\prime}\right) k_{j}}{\sqrt{g_{3}^{k l}\left(\tau^{\prime}\right) k_{k} k_{l}+m^{2}\left(\tau^{\prime}\right) a^{2}\left(\tau^{\prime}\right)}}
$$

where $k_{i}$ are the spatial components of the conserved particle momentum, $q_{i}$ is the initial position, and $g_{3}^{i j}\left(\alpha_{1}(\tau), \alpha_{2}(\tau)\right)$ is the inverse of the Kasner spacespace metric appearing inside the square brackets in Eq. (2).

With this expression, Eq. (8), and noting that the integral converges for all physical parameters of the fields that determine the spacetime $\left(p_{1,2,3}, T_{1,2,3}, g_{p}, \rho_{r}\right)$ and all parameters of the geodesics $\left(k_{1,2,3}, q^{1,2,3}\right)$, we are effectively done with the proof of geodesic completeness. We stress that our complete solutions for $\alpha_{1,2}$ and the gauge invariant combination $a(\tau) m(\tau)=g_{p} a_{E}(\tau) h_{E}(\tau)=$ $g_{p} h_{\gamma}(\tau)$ are continuous and sufficiently well-behaved at 
the crunch and bang; this insures that the geodesics constructed from them are also continuous. The fact that the Weyl curvature and the Weyl-invariant quantities discussed in Refs. 18 19] diverge does not affect this conclusion one iota. This is clearly exemplified in Fig. 1 where we have chosen a typical geodesic and computed the behavior of the geodesic component $x^{3}(\tau)$ and the proper speed. Both are continuous throughout the big crunchbig bang transition and the proper speed is bounded by the speed of light, all despite the fact that the Weyl curvature and other quantities diverge and the Weyl-invariant metric is singular. Even though we do not expect to find a coordinate system to remove all curvature singularities, these are not sufficiently severe in our case to prevent the geodesics from completing their cosmic journeys.

We emphasize that this note pertains to all the solutions of the field equations in the vicinity of the cosmological singularities and all the geodesics in those geometries, as obtained using our proposal ${ }^{23}$ This includes both massive and massless particles.23 (In our general geodesic expression Eq. (8) that is expressed in terms of momenta, all that is needed to cover the case of lightlike geodesics is to set the mass or Higgs coupling $g_{p}$ to zero. As is well known, this can also be obtained from the particle action in Eq. (7) by first defining the canonical momenta and keeping the momenta fixed while taking the zero mass limit; or, equivalently, rewriting the action Eq. (7) in the first order formalism and then taking the limit.)

Our central point is that the continuation of the geometry beyond the singularity is established because we have shown in our case that all geodesics go through the relevant singularities. Classically, this geodesic completeness is not affected by the divergent curvatures that we ${ }^{24}$ and others have identified. ${ }^{23}$ In fact, the completion of the geometry is not supposed to eliminate the curvature singularities. Rather, it is supposed to show that, despite the curvature singularities, physical information can and does journey from cycle to cycle through the cosmological singularities 23. Hence, we can claim the geodesic completeness for all homogeneous cosmological field configurations of the standard model coupled to gravity. Further details and a more thorough discussion of geodesics, geodesic deviation and geodesic completeness in Weylinvariant theories are given in Ref. [25].

Of course, our purely classical analysis does not include strong quantum gravity effects near the singularities because the technology does not yet exist to do those computations ${ }^{23}$ Nevertheless, finding classical geodesical completeness and the complete set of classical solutions is very useful. Having the guidance and physical insight of classical analysis is often a reasonable starting point in understanding quantum theoretic descriptions of physical phenomena, especially when there are indications of new physics, as presented here. For example, suppose one wished to pursue physics near the singularity in the framework of string theory. Our solutions provide the starting point because they provide geodesically complete cyclic background geometries (metric and dilaton) consistent with perturbative worldsheet conformal symmetry as required by the quantization of the string moving in backgrounds. Our classical calculations suggest exciting new phenomena to be explored. Based on historical precedents, it is reasonable to suppose that some or all qualitative features will survive quantum (string) corrections.

We thank A. Ijjas for comments on the manuscript. Research at Perimeter Institute is supported by the Government of Canada through Industry Canada and by the Province of Ontario through the Ministry of Research and Innovation. This research was partially supported by the U.S. Department of Energy under grant number DE-FG03-84ER40168 (IB) and under grant number DEFG02-91ER40671 (PJS).
1 A. H. Guth, Phys. Rev. D23, 347 (1981); A. D. Linde, Phys. Lett. B108, 389 (1982); A. Albrecht, P. J. Steinhardt, Phys. Rev. Lett. 48, 1220 (1982).

2 R. Penrose, Annals N.Y.Acad.Sci. 571, 249 (1989).

${ }^{3}$ G. Gibbons and N. Turok, Phys.Rev. D77, 063516 (2008), hep-th/0609095.

4 I. Bars and S-H. Chen, "The Big Bang and Inflation United by an Analytic Solution ", Pays. Rev. D83 043522 (2011) arXiv:1004.0752.

5 I. Bars, S-H. Chen and N. Turok, Phys. Rev. D84, 083513 (2011) arXiv:1105.3606.

6 I. Bars, "Geodesically Complete Universe", in Proceedings of the DPF- 2011 Conference, Providence, RI, August 8-13, 2011, arXiv:1109.5872.

7 I. Bars, S-H. Chen, P. J. Steinhardt and N. Turok, Phys. Lett. B715, 278 (2012) arXiv:1112.2470.

${ }^{8}$ I. Bars, S-H. Chen, P. J. Steinhardt and N. Turok, Phys. Rev. D86, 083542 (2012) arXiv:1207.1940.

9 I. Bars, "Traversing Cosmological Singularities, Com- plete Journeys Through Spacetime Including Antigravity," (2012) arXiv:1209.1068.

10 I. Bars, P.J. Steinhardt, N. Turok, (2013) "Local conformal symmetry in physics and cosmology," arXiv:1307.1848.

11 I. Bars, P.J. Steinhardt, N. Turok, Phys. Lett. B726, 50 (2013).

12 P. J. Steinhardt and N. Turok, Science 296, 1436 (2002).

13 P. J. Steinhardt and N. Turok, Phys. Rev. D65, 126003 (2002).

14 A. Starobinsky, Pis'ma Astron. Zh., volume 7, 67-72, 1981.

15 T. Futamase, T. Rothman and R. Matzner, Phys. Rev. D 39, 405 (1989).

16 L. R. Abramo, L. Brenig, E. Gunzig and A. Saa, Phys. Rev. D 67, 027301 (2003) gr-qc/0210069.

17 P. Caputa, S. S. Haque, J. Olson and B. Underwood, Class. Quant. Grav. 30, 195013 (2013) arXiv:1306.0012 [hep-th]].

18 J.M. Carrasco, W. Chemissany, and R. Kallosh (2013), arXiv:1311.3671

19 R. Kallosh and A. Linde (2013), arXiv:1311.3326. 
20 L. Anderson and A. D. Rendall, Comm. Math. Phys., 218, 479 (2001).

21 C. W. Misner, Phys. Rev. Lett. 22 (1969) 1071.

22 V. A. Belinsky, I. M. Khalatnikov, E. M. Lifshitz, Adv. Phys. 19525 (1970).

${ }^{23}$ In a Note Added in Proof in Ref. 18 written after our preprint, the authors introduce new arguments (and repeat some past ones). However, all new points (as well as old ones) were already addressed in our original preprint and earlier papers. For clarity, though, we have edited our concluding remarks and marked with this footnote to in- dicate where the direct response to each new point can be found.

24 The presence of singularities in components of the curvature tensor $R_{\mu \nu \lambda \sigma}$ were well known to us (see e.g. paragraph following Eq.(6) in Ref. ${ }^{9}$ ) but we understood that these do not prevent the completion of the geometry; hence our emphasis on geodesically complete geometries all along in all of our papers [5-11].

25 I. Bars, P.J. Steinhardt, N. Turok, "Geodesic completeness and the Higgs," in preparation. 